\title{
EXCLUSIVE USE OF ARTERIAL GRAFTS IN CORONARY ARTERY BYPASS OPERATIONS FOR THREE-VESSEL DISEASE: USE OF BOTH THORACIC ARTERIES AND THE GASTROEPIPLOIC ARTERY IN 256 CONSECUTIVE PATIENTS
}

Jan G. Grandjean, MD

Adriaan A. Voors, MD

Piet W. Boonstra, MD, PhD

Peter den Heyer, MD, PhD

Tjark Ebels, MD, PhD

\begin{abstract}
Methods: From September 1989 to September 1994 we operated on a consecutive group of 256 patients with three-vessel disease in whom we used the right gastroepiploic artery together with both internal thoracic arteries. Vein grafts were not used in these patients. This population consisted of 233 men and 23 women whose ages ranged from 31 to 77 years (mean age 57.8 years). Results: Hospital morbidity and mortality were not directly related to the use of the gastroepiploic artery. Patency of the anastomoses in a subgroup of 56 patients $(22 \%)$ a mean of 16 months after the operation was $\mathbf{9 8 \%}$ for the left internal thoracic artery, $96 \%$ for the right internal thoracic artery, and $\mathbf{8 8 \%}$ for the gastroepiploic artery. Five-year actuarial survival (including in-hospital deaths) was $95.9 \%$ and was related only to age. From discharge until the end of follow-up, two patients had a myocardial infarction, six patients underwent a reintervention procedure, and 18 patients had a return of angina pectoris. Conclusion: We conclude that the concomitant use of the gastroepiploic artery with the both internal thoracic arteries has low morbidity and mortality in patients with three-vessel disease operated on by experienced surgeons. At this moment, we have no reason to believe graft patency will deteriorate in the future. On the basis of these results, the knowledge that arteries are to be preferred over veins for coronary bypass grafting, and the absence of a leg incision, we believe this operative technique is superior to the use of venous grafts. ( $J$ Thorac Cardiovasc Surg 1996;112:935-42)
\end{abstract}

T he use of the internal thoracic artery (ITA) graft for myocardial revascularization is superior to that of saphenous vein grafts because of higher patency rates and a lower mortality and morbidity. ${ }^{1-4}$ Several authors recently showed that the use of both the left and right ITAs further decreases recurrence of angina and reoperations. ${ }^{5-8}$ Still, venous grafts continue to be used in the majority of patients having coronary bypass operations, mainly because of a shortage of arterial material and the

From the Thoraxcenter, University Hospital Groningen, Groningen, The Netherlands.

This study was funded by the J. K. de Cock Foundation.

Received for publication Dec. 20, 1996; revisions requested Feb. 6, 1996; revisions received March 11, 1996; accepted for publication May 8, 1996.

Address for reprints: Jan G. Grandjean, MD, Thoraxcenter, Department of Cardiothoracic Surgery, University Hospital of Groningen, Hanzeplein 1, 9713 EZ Groningen, The Netherlands.

Copyright (c) 1996 by Mosby-Year Book, Inc.

$0022-5223 / 96 \$ 5.00+0 \quad 12 / 1 / 74704$ inability to reach the inferior surface of the heart with ITAs. ${ }^{9}{ }^{10}$ In recent years, the gastroepiploic artery (GEA) has been a workable alternative to the saphenous vein graft, with short-term patency and clinical outcome similar to those of ITA grafts. ${ }^{11-17}$

We report on a series of 256 consecutive patients with three-vessel disease having coronary bypass operations with the use of arterial grafts exclusively; both the right and the left ITAs and the GEA were used, and venous grafts were not used. The aims of this study were to describe operative results, as well as midterm angiographic and clinical outcomes, of this homogeneous group and to identify risk factors that influenced these results.

\section{Patients and methods}

Patient population. Between September 1989 and September 1994, 3720 patients were operated on for coronary artery disease in our institution. Revascularization of all three vessels was performed in 1495 patients. From this group of patients, venous grafts exclusively were used in 104 patients $(7 \%)$. A single ITA in conjunction with venous grafts was used in 1135 patients $(76 \%)$ and the 
GEA in combination with one or two ITAs was used in 256 patients $(17 \%)$. The study population consisted of these 256 consecutive patients with three-vessel disease in whom the GEA was used in combination with both ITA grafts but no venous grafts. The main reason that only arterial grafts were used was that three of the eight surgeons who performed 244 of the 256 operations preferred this approach. Other indications also played a role, such as the lack of other graft material and young age of some of the patients. In the study period, these three surgeons used this approach in $48 \%, 36 \%$, and $13 \%$ of their patients in whom three vessels were bypassed. The main contraindications to this form of revascularization were technical problems such as previous major operations in this area or extreme obesity. It was not performed in a few patients with a short life expectancy. In $4 \%$ of the patients in whom we planned to do all-arterial threevessel revascularization, the GEA appeared to be too small for use as a pedicled graft. In half of these patients, the GEA was used as a free graft, and in the other half the surgeon decided not the use the GEA as a graft.

Elective angiography was performed in the 56 patients. In $25 \%$ of these patients, angiography was performed because of the return of symptoms. The other $75 \%$ of the patients were willing to undergo this procedure after giving written informed consent.

All data were updated at the end of 1994 and were gathered by interview and examination by the patient's own cardiologist.

Technique of the operation. The standard median sternotomy was extended $5 \mathrm{~cm}$ caudally, after which the right ITA and then the left ITA were harvested. The pedicles were wrapped in a sponge soaked in papaverine solution. The pleural cavity was intentionally left closed. The peritoneal cavity was opened and the GEA was palpated gently to asses its caliber. The GEA was dissected with the use of two surgical clips (Hemoclip, medium, Pilling Weck, Research Triangle Park, N.C.) on each side branch to the stomach and omentum. The branches were divided by electrocoagulation. The GEA was dissected leftward two thirds of the distance along the great curvature of the stomach and proximal to the pylorus. After systemic heparinization, the distal part of the GEA graft was divided and $0.5 \mathrm{ml}$ of a solution of papaverine $(0.1 \mathrm{mg} / \mathrm{ml})$ was gently injected into the distal part of the graft or was injected into the fatty tissue of the pedicle. The GEA was always routed anterior to the pylorus and the left lobe of the liver to the pericardial cavity. An incision in the diaphragm was made anterior to the inferior vena cava. The coronary anastomoses were made with running 8-0 polypropylene sutures. The pedicle was fixed to the epicardium by means of two or three sutures (6- 0 polypropylene) to avoid kinking of the graft at the anastomosis. The peritoneum was closed with a running resorbable suture without a drain.

The right ITA was tunneled under the mediastinal fat, directly over the superior vena cava by incision of the pericardium, and was routed through the transverse sinus to reach the circumflex coronary artery. To reach the left anterior descending coronary artery the right ITA was also tunneled under the fatty tissue to avoid fixation to the sternum and was routed over the aorta. The left ITA was also routed under the mediastinal fat, deep along the parietal left pleura to either the left anterior descending or the circumflex coronary artery. Postoperative anticoagulation treatment consisted of acetyl salicylic acid $100 \mathrm{mg}$ daily.

Technique of angiography. Elective follow-up angiography was performed with the Judkins technique through the femoral approach. Standard Judkins $7 F$ left and right coronary catheters, as well as pigtail catheters, were used for the native coronary artery injections and the left ventricular angiogram. ITA catheters were used for selective angiography of the left and right ITA grafts. A special technique was developed for angiography of the GEA graft. The celiac trunk was cannulated by means of a lateral projection with a $7 F$ special GEA catheter of our own design (built to specification by Cordis Europe NV, Roden, The Netherlands). Using the posteroanterior view, we advanced a soft, steerable 0.035 -inch guide wire (Terumo Corporation, Tokyo, Japan) into the common hepatic artery or subselectively into the gastroduodenal artery. The contrast catheters were advanced over these guide wires into the gastroduodenal artery or even into the origin of the GEA. This technique allowed for selective hand injections of contrast medium and for excellent visualization of the GEA graft and its anastomoses, as well as the native coronary artery distal to this anastomosis with multiple projections. All angiograms were recorded on movie film at 12.5 to 25 frames per second and were reviewed by two independent observers.

End points. 1. Progression of atherosclerosis (graft stenosis) on cardiac catheterization was defined as any visible stenosis anywhere in the graft(s), including the sites of anastomoses. A patent graft was defined as a nonstenotic graft.

2. In-hospital complications were defined as any untoward postoperative medical event that occurred during hospitalization. Atrial fibrillation was analyzed separately from other in-hospital complications.

3. In-hospital and follow-up data were gathered on death, myocardial infarction, reintervention (percutaneous transvenous coronary angioplasty or coronary artery bypass grafting), or the return of angina pectoris.

Statistical analysis. The possible influence of the following factors on clinical outcome of our study population was analyzed: age; sex; history of angioplasty, bypass grafting, myocardial infarction, diabetes mellitus, hypertension, hypercholesterolemia, cholecystectomy, or laparotomy; body mass index; preoperative angina pectoris (New York Heart Association class); duration of operation; number of distal anastomoses; surgeon; use of free grafts; and location of anastomosis of the GEA.

Univariate and multivariate analysis of factors predicting graft progression, postoperative complications, and atrial fibrillation. The $\chi^{2}$ test was used for calculations of associations in contingency tables. When the sample was not large and the expected values were less than 5, we used Fisher's exact test. For differences between means, the $t$ test was used. When variances where unequal, the MannWhitney $U$ test was used. Factors that reached a significance level of less than 0.1 or any other clinically important factor such as age and sex were included for multivariate logistic regression analysis. The final model 
Table I. Preoperative patient characteristics

\begin{tabular}{lrc}
\hline & No. & Percent of 256 \\
\hline NYHA & & \\
Class II-III & 173 & 67 \\
class IV & 83 & 33 \\
Myocardial infarction & 140 & 54 \\
More than one infarction & 20 & 7.8 \\
Hypercholesterolemia & 133 & 52 \\
Diabetes & 13 & 5.0 \\
PTCA & 26 & 10 \\
Reoperation & 28 & 11 \\
Prior laparotomy (upper) & 9 & 3.6 \\
\hline
\end{tabular}

NYHA, New York Heart Association; PTCA, percutaneous transluminal coronary angioplasty.

consisted only of factors that had reached a significance level of less than 0.1 in previous models.

Univariate and multivariate survival analysis of death, myocardial infarction, reintervention, or the return of angina pectoris. To identify prognostic factors that might have "explained" outcome differences in our study population, we estimated survival curves by the method described by Kaplan and Meier. ${ }^{18}$ Differences in survival times between groups were calculated by the log-rank and the Wilcoxon tests. All variables with a significance level of $p<0.1$ in at least one of these univariate tests and all other clinically important variables, such as age and sex, were included for multivariate analysis as proposed by Cox. ${ }^{19}$ The final model consisted only of variables that had reached a significance level of less than 0.1 in the previous model. We checked the assumption of proportional hazards for each predictor variable by estimating the plots of the logarithm of the cumulative hazard against the logarithm of time.

\section{Results}

Patients. The study group consisted of 233 men and $23(10 \%)$ women with a median age of 57.8 years (range 31 to 77 years). Preoperative patient characteristics are shown in Table I. These patients were a subgroup from 1495 patients who underwent three-vessel revascularization. This group consisted of 1128 men and 367 women ( $24 \%$ ) with a mean age 65.5 years (range 31 to 87 years).

Grafts and anastomoses. In the group of 256 patients operated on with the use of three arterial grafts, a total of 931 anastomoses (667 ITA anastomoses, 264 GEA anastomoses) were performed; there were 768 arterial grafts and no vein grafts (Table II). The mean number of anastomoses per patient was 3.64. The mean number of anastomoses of left ITA grafts was 1.5 , right ITA grafts 1.1 , and GEA grafts 1.0. A sequential left ITA graft was used in 129 patients $(50 \%$ ) (117 with two distal anastomoses, 12 with three distal anastomoses). A sequen-
Table II. Number of anastomoses of bypass arteries to coronary arteries

\begin{tabular}{lrrr}
\hline & GEA & LITA & RITA \\
\hline LAD & 1 & 160 & 88 \\
Diagonal & 1 & 98 & 13 \\
CX & 6 & 139 & 166 \\
RCA & 41 & - & 3 \\
RPD & 198 & - & - \\
RPL & 17 & - & - \\
Total & 264 & 397 & 270 \\
\hline
\end{tabular}

GEA, Gastroepiploic artery; LITA, left internal thoracic artery; RIMA, right internal thoracic artery; $L A D$, left anterior descending coronary artery; $C X$, circumflex coronary artery; $R C A$, right coronary artery; $R P D$, right posterior descending; $R P L$, right posterolateral.

tial right ITA graft was used in 15 patients $(6 \%)$ and a sequential GEA graft in eight patients (3.1\%). A free ITA graft was used in seven patients and a free GEA graft was used in only three patients.

In-hospital complications. Four patients $(1.8 \%)$ died in the hospital. One patient died of brain infarctions, one patient of a myocardial infarction caused by embolization of atheromatous material from the aorta, one died of an anterior infarction after the left anterior descending branch was not found, and one died of cardiac failure. Two patients underwent reoperation a few hours after the first operation because of ischemia of the inferior aspect of the heart. A vein graft was placed next to the GEA anastomosis in one of the patients. Five postoperative angioplasty procedures were performed; one angioplasty of the right coronary artery, because the GEA was occluded, two angioplasties of the GEA at its anastomosis, and two angioplasties of the left anterior descending coronary artery. Mediastinitis occurred in two patients. The sternum was secondarily closed after a period of open treatment, after 3 and 4 weeks. Mild gastrointestinal bleeding occurred twice and was treated medically. None of the patients had severe late abdominal symptoms at follow-up. Twenty-five patients $(10 \%)$ used $\mathrm{H}^{2}$ blockers. In-hospital myocardial infarction occurred in six patients $(2.4 \%)$ according to clinical symptoms, electrocardiographic changes, and elevated levels of cardiac enzymes. Reoperations for persistent bleeding were necessary in 18 patients $(7.8 \%)$. In two patients the bleeding was from the pedicle of the GEA graft. In nine patients, we needed an intraaortic balloon pump; in three of the nine patients the pump had already been inserted before the operation because of severe stenosis of the left main coronary artery. 


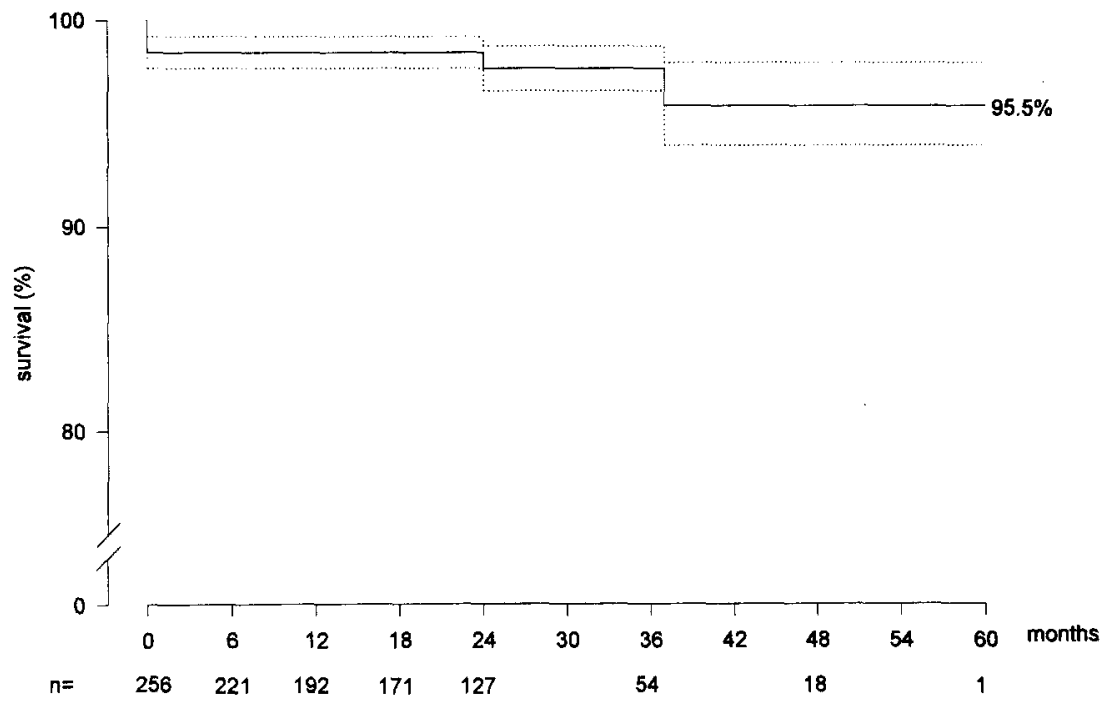

Fig. 1. Five-year actuarial survival (including in-hospital death) for patients who received three arterial grafts.

Table III. Results from multiple logistic regression for identifying risk factors for in-hospital complications, postoperative paroxysmal atrial fibrillation, and graft occlusion on postoperative angiography

\begin{tabular}{|c|c|c|c|c|}
\hline & Yes & No & Odds ratio $(95 \% \mathrm{CI})$ & $p$ Value \\
\hline \multicolumn{5}{|l|}{ In-hospital complications } \\
\hline Duration of operation (mean) & $243 \min$ & $213 \mathrm{~min}$ & $1.008(1.002-1.013)$ & 0.004 \\
\hline \multicolumn{5}{|l|}{ Atrial fibrillation } \\
\hline Age (mean) & 62.8 years & 56.9 years & $1.073(1.029-1.120)$ & 0.001 \\
\hline Hypercholesterolemia & $36 \%$ & $59 \%$ & $0.47(0.22-0.97)$ & 0.042 \\
\hline Use of aprotinin & $30 \%$ & $14 \%$ & $2.29(1.05-2.93)$ & 0.049 \\
\hline \multicolumn{5}{|l|}{ Graft occlusion } \\
\hline Duration of operation (mean) & $273 \mathrm{~min}$ & $223 \min$ & $1.014(1.001-1.026)$ (per min) & 0.033 \\
\hline
\end{tabular}

Only risk factors with $p<0.05$ are presented. Data are presented as odds ratios and $95 \%$ confidence intervals $(C I)$.

In summary, in-hospital complications occurred in $48(19 \%)$ of the patients. Both univariate and multivariate analysis revealed that the in-hospital complications were individual surgeon-dependent $(p=0.002)$. After adjustment for this and other clinically important covariates in a multivariate logistic regression model, only the duration of the operation appeared to be an independent predictor of the occurrence of in-hospital complications (Table III). There was a gradual increase in in-hospital complications with increased duration of the operation, where the odds-ratio per minute was 1.008 (95\% confidence interval [CI] 1.002 to $1.013, p=$ 0.004). This means that (for example) every 30 minutes, the risk for in-hospital complications increased $1.008^{30}=1.27$ times.

Atrial fibrillation. Paroxysmal postoperative atrial fibrillation occurred in 43 patients $(16.8 \%)$ and was in most cases successfully controlled by medication. Again, there appeared to be a correlation between atrial fibrillation and the surgeon who performed the operation. After adjustment for this and other covariates, age appeared to be a strong independent risk factor for postoperative atrial fibrillation (see Table III). The mean age of patients with postoperative atrial fibrillation was 62.8 years, compared with 56.9 years in the group without atrial fibrillation. The multivariate odds ratio per year was 1.073 (95\% CI 1.029 to $1.120, p=0.001$ ). Other independent risk factors were the use of aprotinin (odds ratio 2.3, 95\% CI 1.0 to $5.2, p<0.05$ ) and a decreased risk for patients with a history of hypercholesterolemia (odds ratio $0.5,95 \%$ CI 0.2 to 1.0 , $p=0.04$ ).

Mortality and morbidity during follow-up. No patient was lost to follow-up. Mean postoperative 
Table IV. Results from multivariate Cox proportional hazards analysis for identifying risk factors for death, myocardial infarction, and the return of angina pectoris

\begin{tabular}{llcc}
\hline & Risk factor & Hazard ratio (95\% CI) & $p$ Value \\
\hline Death & Age & $1.52(1.18-1.95)$ & 0.001 \\
Myocardial infarction & Redo operation & $6.3(1.5-26.5)$ & 0.01 \\
Angina pectoris & History of PTCA & $4.6(1.4-15.2)$ & 0.01 \\
& Female sex & $4.6(1.3-16.3)$ & 0.02 \\
\hline
\end{tabular}

Only risk factors with $p<0.05$ are presented. Data are presented as hazard ratios and $95 \%$ confidence intervals $(C I)$. PTCA, Percutaneous transluminal coronary angioplasty.

Table V. Three-year actuarial survival for myocardial infarction, reintervention, or the return of angina pectoris, for a group of patients older than 65 and 70 years, respectively, compared with patients younger than 65 and 70 years, respectively: $p$ values are calculated by the log-rank test

\begin{tabular}{lcccccc}
\hline \multicolumn{1}{c}{ Survival for: } & $<65 y r(\%)$ & $\geq 65 y r(\%)$ & $p$ Value & $<70 y r$ & $\geq 70 y r$ & $p$ Value \\
\hline Myocardial Infarction & 96 & 95 & 0.18 & 96 & 95 & 0.30 \\
Reintervention & 95 & 97 & 0.59 & 95 & 100 & 0.23 \\
Angina pectoris & 90 & 88 & 0.65 & 89 & 90 & 0.91 \\
\hline
\end{tabular}

follow-up was $25 \pm 15$ months (up to 58 months). During follow-up, six patients died, four of whom are described in the section In-hospital complications. The other two patients died 25 and 38 months after the operation, one of rupture of an abdominal aortic aneurysm and the other of a noncardiovascular cause. Five-year actuarial survival (including in-hospital death) for this group of patients who received three arterial grafts was $95.9 \%$ (Fig. 1). From discharge to the end of follow-up, two patients had a myocardial infarction, six patients underwent a reintervention procedure, and 18 patients had a return of angina pectoris. Three-year actuarial freedom from any of these events (including in-hospital events) was $85 \%$.

Multivariate Cox proportional hazards analysis revealed that age was an independent risk factor for death (hazard ratio per year $1.5,95 \%$ CI 1.2 to 1.9 , $p=0.001)$. The only independent risk factor for myocardial infarction was reoperation (hazard ratio $6.3,95 \%$ CI 1.5 to $26.5, p=0.01$ ), and we found no independent risk factor for reintervention. Female patients were significantly more at risk for the return of angina pectoris (hazard ratio $4.6,95 \%$ CI 1.3 to $16.1, p=0.02$ ), and the return of angina pectoris was also significantly associated with a previous angioplasty procedure (hazard ratio $4.6,95 \%$ CI 1.4 to $15.2, p=0.01$ ) (Table IV).

Older patients did not have increased risks for myocardial infarction, reintervention, or the return of angina pectoris during follow-up. Actuarial survival analysis yielded comparable results between
Table VI. Patency rate per anastomosis according to grafted coronary artery

\begin{tabular}{lccc}
\hline & $L A D$ & $C X$ & $R C A$ \\
\hline LITA & $53 / 54$ & $33 / 33$ & \\
RITA & $25 / 25$ & $30 / 32$ & $2 / 2$ \\
GEA & & $3 / 5$ & $49 / 54$ \\
\hline
\end{tabular}

LITA, Left internal thoracic artery; RMA, right internal thoracic artery. $G E A$, gastroepiploic artery; $L A D$, left anterior descending artery; $C X$, circumflex artery; $R C A$, right coronary artery.

patients older and younger than 65 years, as well as between patients older and younger than 70 years (Table V). As can be expected, older patients were at greater risk of dying during follow-up. Five-years survival for patients older than 65 years was $89 \%$, compared with $98 \%$ for patients younger than 65 years $(p=0.04)$.

Graft patency. Angiography was performed in 56 patients after a mean of 16 months (up to 61 months). In $25 \%$ of these patients, angiography was performed because of the return of symptoms and in the other group for study reasons only.

In 49 patients (88\%), the GEA with its anastomoses were still patent, whereas a patent left ITA was present in 55 patients $(98 \%)$ and a patent right ITA in 54 patients $(96 \%)$ (Table VI). There was no difference in patency whether the left or right ITA was anastomosed to the left anterior descending or the circumflex artery. Even the anastomoses between the GEA and right coronary artery or its branches had a patency rate of $90 \%$. However, GEA anastomoses to the circumflex artery appeared to be 
patent at angiographic follow-up in only three of the five patients $(60 \%)$ in whom the GEA was used.

In multiple logistic regression analysis, only duration of operation remained a significant predictor for total progression of disease at follow-up angiography (see Table III). We found no specific predictive factors for progression in the GEA and its anastomoses.

\section{Discussion}

Several authors have reported that long-term survival after coronary bypass operations with the use of one ITA graft to the left anterior descending coronary artery is superior to the use of autologous saphenous vein grafts, particularly in younger patients. ${ }^{3,20-22}$ Moreover, the use of both the left and right ITAs further improved short-term and midterm morbidity and mortality, although long-term outcome in patients receiving two ITAs remains unknown. ${ }^{5-7}$ In our institution, an increasing number of second operations are performed on patients who were previously operated on with a combination of one ITA and one vein graft, in whom the vein grafts closed but the ITA is patent. On the basis of these findings, it seemed desirable to use arterial grafts exclusively in patients with three-vessel disease. However, complete arterial revascularization for three-vessel disease usually cannot be achieved with ITAs alone, because too many anastomoses are needed for only two grafts. Another shortcoming of both left and right ITAs is their anatomic position, which often prevents revascularization of the posterior surface of the heart. Finally, a leg incision for harvesting the saphenous vein graft is usually an important source of discomfort to patients. These findings together emphasized the need to identify additional arteries suitable for myocardial revascularization.

The right GEA was found to be a promising alternative bypass conduit for the saphenous vein. ${ }^{11-13}$ Large series of patients reported on by Suma and associates ${ }^{14}$ and by us ${ }^{16}$ showed that the in situ GEA has a suitable diameter for bypass grafting and is long enough to reach coronary arteries on the inferior ventricular wall; anastomoses to the left anterior descending and circumflex arteries were also possible. These studies also showed good short-term and midterm patency and a low mortality and morbidity. However, these patient groups also consisted of patients with one- or twovessel disease.

Like Jegaden and colleagues, ${ }^{17}$ who had a large series, we also report on a consecutive group of patients in whom the GEA and both ITAs were routinely used for complete arterial revascularization of three-vessel disease. However, patients with this all-arterial revascularization were younger than the total group of 1495 patients with three-vessel revascularization and consisted of more male patients. Obviously, this technique was preferable in younger patients with a longer life expectancy. On the other hand, older age was not a risk factor for postoperative morbidity and increased graft failure, as demonstrated with coronary angiography.

The use of the GEA in our study group did not lead to a significant increase in perioperative mortality. Overall, four patients $(1.7 \%)$ died in the hospital, and mortality was not related to an inferior wall infarction, the area revascularized by the GEA. At late follow-up neither of the two deaths was cardiac related. In addition, use of the GEA did not lead to an increase in perioperative morbidity for coronary bypass operations. Reoperations for persistent bleeding were performed in 18 patients. This prevalence of bleeding complications was probably due to the use of antithrombotic therapy until the day before the operation and to the cautious use of aprotinin. Only three of these 18 reoperations for persistent bleeding were due to bleeding of the gastroepiploic pedicle, located in the pericardial and abdominal cavities. Gastric morbidity was extremely limited. In this group we observed no gastric complications after harvesting the GEA. Within this group of patients, the duration of the operation appeared to be an independent predictor for the occurrence of postoperative in-hospital complications and for graft occlusion during follow-up. Dissection of both ITAs and of the GEA is done by the same surgeon, who does the entire operation from skin to skin. However, we agree with Lytle and coworkers $^{13}$ that the use of the GEA increases the difficulty and complexity of the operation. The operation should be performed by experienced surgeons.

A subgroup of patients underwent follow-up angiography at a mean of 16 months after the operation. It should be noted, however, that $25 \%$ of these patients underwent coronary angiography because of the return of symptoms. Therefore this was a biased population, in which an increased occlusion rate could be expected. The GEA was widely patent in $88 \%$ of the patients, however, despite an occlusion rate of $40 \%$ when the GEA was used as a graft for the circumflex coronary artery. The latter finding 
could be explained by the need for a longer graft to the circumflex artery and thus a diminished diameter of the GEA. Therefore the diameters of the distal anastomoses to the circumflex artery were probably much smaller than when the GEA was used for branches of the right coronary artery. Despite this increased occlusion rate, the use of the GEA for the circumflex artery in the entire study population did not appear to be a risk factor for mortality or morbidity during follow-up.

The most common postoperative complication was atrial fibrillation. Independent risk factors for atrial fibrillation were age, no history of hypercholesterolemia, and the use of aprotinin. Age is a widely known risk factor for atrial fibrillation, but we could not explain the other risk factors. The risk for myocardial infarction during midterm follow-up was greater after reoperations. Independent predictive factors for the return of angina pectoris were a previous coronary angioplasty procedure before the operation and female sex. The latter probably results from the fact that the grafts and grafted arteries are smaller in women than in men. ${ }^{23}$ Age appeared to be no risk factor for in-hospital complications and morbidity during midterm follow-up (see Table V). Overall, in-hospital complications, prevalence of myocardial infarction, reintervention, and the return of angina pectoris did not seem to be different between patients with all-arterial revascularization and those with a standard bypass operation in which one ITA graft and vein grafts were used.

Comparison has to be made between the saphenous vein and the GEA as revascularization conduits. Unlike the saphenous vein graft, the GEA was found to have characteristics comparable with those of the ITAs. O'Neil and associates ${ }^{24}$ concluded from their study on endothelial function of the GEA that the artery possessed the necessary properties to be a potentially successful arterial bypass graft. $\mathrm{We}^{25}$ previously reported similar relaxation of GEA and ITA to methacholine and sodium nitrite, suggesting comparable endothelial function in the two arteries. This implies that the protective role of the endothelium, which probably contributes to the high patency rate of ITAs, is also applicable to GEAs.

Our choice for routine use of the GEA rather than other arterial grafts, such as the radial artery or inferior epigastric artery, is fueled by the ease of harvesting, the advantage of an in situ graft, and its flow and size. There is also no need for an extra skin incision.
Encouraged by these results, we now use the GEA graft as the first adjunct to both ITAs even in elderly patients. Previous gastric resection is an absolute contraindication to the use of the GEA as a graft, whereas severe obesity, abdominal aortic operations, and previous cholecystectomy are minor considerations. In patients with insulin-dependent diabetes the use of two ITA grafts was associated with a higher risk of sternal infections. ${ }^{26}$ However, we performed these operations in 11 patients with insulin-dependent diabetes and observed no problems with sternal wound healing.

In conclusion, our patients with three-vessel coronary artery disease who were revascularized with the GEA and the left and right ITAs had excellent short-term and midterm results. Mortality and morbidity were low even in elderly patients. At this time, we have no indications of progressive deterioration of graft patency. On the basis of these results, together with the knowledge that arteries are to be preferred over veins for coronary bypass grafting and the absence of a leg incision, we believe this operative technique to be superior to the use of venous grafts.

\section{REFERENCES}

1. Lytle BW, Loop FD, Cosgrove DM, Ratliff NB, Easley K, Taylor PC. Long-term (5 to 12 years) serial studies of internal mammary artery and saphenous vein coronary bypass grafts. J Thorac Cardiovase Surg 1985;89:248-58.

2. Singh RN, Sosa JA, Green GE. Long-term fate of the internal mammary artery and saphenous vein grafts. J Thorac Cardiovase Surg 1983;86:359-63.

3. Loop FD, Lytle BW, Cosgrove DM, et al. Influence of the internal mammary artery graft on 10-year survival and other cardiac events. N Engl J Med 1986;314:1-6.

4. Acinapura AJ, Rose DM, Jacobowitz IJ, et al. Internal mammary artery bypass grafting: influence on recurrent angina and survival in 2,100 patients. Ann Thorac Surg 1989;48:186-91.

5. Lytle BW, Cosgrove DM, Loop FD, et al. Perioperative risk of bilateral internal mammary artery grafting: analysis of 500 cases from 1971 to 1984 . Circulation 1986;74(Suppl):III3741.

6. Fiore AC, Naunheim KS, Dean P, et al. Results of internal thoracic artery grafting over 15 years: single versus double grafts. Ann Thorac Surg 1990;49:202-9.

7. Dion R, Etienne PY, Verhelst R, et al. Bilateral mammary grafting: clinical, functional and angiographic assessment in 400 consecutive patients. Eur J Cardiothorac Surg 1993;7: $287-94$.

8. Van Sterkenburg SMM, Ernst SMPG, de la Rivière AB, Defauw JAM, Hamerlynck RPHM, Knaepen PJ, et al. Triple sequential grafts using the internal mammary artery: an angiographic and short-term follow-up study. J Thorac Cardiovasc Surg 1992;104:60-5. 


\section{Grandjean et al.}

9. Grondin CM, Campeau L, Thornton JC, Engle JC, Cross FS, Schreiber H. Coronary artery bypass grafting with saphenous vein. Circulation 1989;79(Suppl):I24-9.

10. Bourassa MG. Fate of venous grafts: the past, the present, and the future. J Am Coll Cardiol 1991;17:1081-3.

11. Suma $H$, Fukumoto $H$, Takeuchi A. Coronary artery bypass grafting by utilizing in situ right gastroepiploic artery: basic study and clinical application. Ann Thorac Surg; 1987;44;394-7.

12. Mills NL, Everson CT. Right gatroepiploic artery: a third arterial conduit for coronary artery bypass. Ann Thorac Surg 1989;47:706-11.

13. Lytle BW, Cosgrove DM, Ratliff NB, Loop FD. Coronary artery bypass grafting with the right gastroepiploic artery. $\mathrm{J}$ Thorac Cardiovasc Surg 1989;97:826-31.

14. Suma H, Wanibuchi Y, Terada Y, Fukuda S, Takayama T, Furuta S. The right gastroepiploic artery graft: clinical and angiographic midterm results in 200 patients. J Thorac Cardiovase Surg 1993;105:615-23.

15. Manapat AE, McCarthy PM, Lytle BW, Taylor PC, et al. Gastroepiploic and inferior epigastric arteries for coronary artery bypass: early results and evolving applications. Circulation 1994;90(Suppl):II144-7.

16. Grandjean JG, Boonstra PW, Den Heyer P, Ebels T. Arterial revascularization with the right gastroepiploic artery and internal mammary arteries in 300 patients. J Thorac Cardiovasc Surg 1994;107:1309-16.

17. Jegaden O, Eker A, Montagna P, et al. Risk and results of bypass grafting using bilateral internal mammary and right
The Journal of Thoracic and

Cardiovascular Surgery

October 1996 gastroepiploic arteries. Ann Thorac Cardiovasc Surg 1995; 59:955-60.

18. Kaplan E, Meier P. Nonparametric estimation from incomplete observations. J Am Stat Assoc 1958;53:457-81.

19. Cox DR. Regression models and life tables. J R Stat Soc 1972;34:187-202.

20. Cameron AAC, Green GE, Brogno DA, Thornton J. Internal thoracic artery grafts: 20-year clinical follow-up. J Am Coll Cardiol 1995;25:188-92

21. Green GE, Cameron A, Goyal A, et al. Five-year follow-up of microsurgical multiple internal thoracic artery grafts. Ann Thorac Surg 1994;58:74-9.

22. Sergeant P, Lesaffre E, Flameng W, Suy R. Internal mammary artery: methods of use and their effect on survival after coronary bypass surgery. Eur J Cardiothorac Surg 1990;4:72-8.

23. O'Connor GT, Morton JR, Diehl MJ, et al. Differences between men and women in hospital mortality associated with coronary artery bypass graft surgery. Circulation 1993;88:12104-10.

24. O'Neil GS, Chester AH, Allen SP, Luu TN, Tadjkarimi S, Ridley $\mathrm{P}$, et al. Endothelium function of human gastroepiploic artery: implication for its use as a bypass graft. J Thorac Cardiovasc Surg 1991;102:561-5.

25. Buikema $\mathrm{H}$, Grandjean JG, Broek vd S, Gilst W, et al. Differences in vasomotor control between gastroepiploic and left internal mammary artery. Circulation 1992;86(Suppl):II205-9.

26. Kouchoukos NT, Wareing TH, Murphy SF, et al. Risk of bilateral internal mammary artery bypass grafting. Ann Thorac Surg 1990;49:210-9. 